\title{
Palliative care in Parkinson's disease: review of needs assessment tools
}

\author{
Edward W. Richfield ${ }^{1}$, Miriam J. Johnson ${ }^{2}$ \\ ${ }^{1}$ Department of Medicine, Southmead Hospital, Southmead Road, Bristol BS10 5NB, UK; ${ }^{2}$ Wolfson Palliative Care Research Centre, University of \\ Hull, Hull HU6 7RX, UK \\ Contributions: (I) Conception and design: All authors; (II) Administrative support: MJ Johnson; (III) Provision of study materials or patients: All \\ authors; (IV) Collection and assembly of data: EW Richfield; (V) Data analysis and interpretation: All authors; (VI) Manuscript writing: All authors; \\ (VII) Final approval of manuscript: All authors. \\ Correspondence to: Dr. Edward Richfield, MBChB, PhD. Consultant Geriatrician, Department of Medicine, Southmead Hospital, Southmead Road, \\ Bristol BS10 5NB, UK. Email: Edward.richfield@nbt.nhs.uk.
}

\begin{abstract}
Palliative care in Parkinson's disease (PD) has developed significantly in the past five years. The presence of unmet palliative care need, similar to that in malignant conditions, is well established, but may not always be identified by clinicians in everyday practice. To address this, clinicians must be able to rapidly identify, triage, assess and address unmet needs and monitor response to intervention. This is also central to establishing integrated models of care for PD. Systematic evaluation of need can be facilitated by structured assessment tools, and several such tools have now been described for PD. This article reviews the palliative care assessment tools which have been developed for PD, both for patients and also for caregivers. It sets out the psychometric properties of these tools and describes the way in which they may be applied within an overall, systematic assessment of unmet need.
\end{abstract}

Keywords: Palliative care; Parkinson's disease (PD); needs assessment

Submitted Aug 13, 2019. Accepted for publication Sep 19, 2019.

doi: 10.21037/apm.2019.09.18

View this article at: http://dx.doi.org/10.21037/apm.2019.09.18

\section{Introduction}

Palliative care for Parkinson's disease (PD) has developed significantly over the past five years. It is established that people living with $\mathrm{PD}$, in the latter stages of the disease, face similar challenges, with an equivalent palliative care need, to those with malignant conditions $(1,2)$.

In line with the WHO definition (3) and as recommended by the National Institute for Health and Care Excellence (NICE) in the UK (4), palliative interventions may be appropriate even from the time of diagnosis, alongside other active medical interventions, for example help with information management (5) and access to and support with advance care planning (ACP) (6).

\section{Why measure palliative care needs in PD?}

In practice, equitable access to palliative care for People with Parkinson's ( $\mathrm{P} w \mathrm{P})$ based on need, depends on integrated models of care delivery (7). This combines a palliative approach to care, delivered by non-palliative specialists as part of usual practice and access to specialist palliative care (SPC) services on the basis of need (8). This model mirrors approaches to palliative provision for other non-malignant conditions and is based upon effective identification and triage of unmet care needs, by nonpalliative specialists.

Tools and measures which support assessment and documentation of both prevalence of unmet needs and response to intervention in routine clinical care are available. These help us judge the benefit of needs assessment, can drive year on year improvement in clinical outcomes and contribute to service development (9).

Within an integrated system of care different methods for assessing unmet need will be appropriate at different 
times. For example, detailed quantitative assessment may not be feasible or desirable in everyday PD clinic, where a faster screening approach may be preferred. Whereas, in SPC clinic a quantifiable measure of need may be extremely useful to assess change over time and response to treatment. Thus, different palliative assessment tools may be required at various points in the patient journey.

\section{What do we mean by needs assessment?}

A needs assessment tool has been defined as that which provides a consistent and comprehensive system to prompt discussion of a patients' range of support and care needs; helps professionals triage tailored action and is useful for audit and service planning (10). This is based on the recognition that systematic assessment has advantages over usual medical consultation or open-ended questions, facilitating the identification of greater number of symptoms and increased recognition of distressing symptoms (11). Such assessment should not focus solely on medical issues, but embrace a holistic model of care, considering spiritual, psychological and existential needs.

In other conditions, this integrated approach to palliative care has been shown to improve patient outcomes $(12,13)$ and studies have identified a number of general palliative care assessment tools which allow a systematic review of need for patients with cancer $(10)$, or in primary care $(12,14)$. Whilst some tools from the latter group have captured $\mathrm{PwP}$ within their study populations $(15,16)$, they are targeted at general primary care populations and in this article we focus on those tools which have been developed specifically for PwP.

Assessment of palliative care needs in PD may usefully be conceptualised in several steps:

(I) Recognition of those most at greatest risk of unmet needs;

(II) Identification of unmet needs;

(III) Assessment of unmet needs (may include quantification);

(IV) Assessment of change over time and response to intervention.

Identifying those at greatest risk of unmet need is important in order to focus resource effectively. There is no agreed strategy for when best to apply palliative needs assessment in $\mathrm{PD}$, but a growing number of studies have identified factors associated with poor outcome $(17,18)$. Anecdotally, within our own practice, the presence of formed visual hallucinations, onset of Hoehn and Yahr
$(\mathrm{H}+\mathrm{Y})$ stage 3 symptoms and recurrent falls are particular triggers for early consideration of unmet needs, with the aim of engaging in this process before significant cognitive impairment is established, to optimise patient autonomy and joint decision making.

\section{Type of assessment tool}

\section{Respondent}

In selecting a needs assessment tool, it is important to consider the primary respondent. Patient reported outcome measures (PROM) have the obvious advantage of directly assessing patient concerns but may be less useful where cognitive impairment is prevalent; a particular challenge in PD $(19,20)$. Here proxy representation of need may be an attractive alternative, although caution is needed interpreting proxy reported need (21).

\section{Health related quality of Life (HRQoL)}

There are a number of well-established HRQoL tools in PD. While HRQoL is an important measure of wellbeing, it is distinct from needs assessment which aims to identify the most important concerns for the individual and which should be priority therapeutic targets (22).

\section{Setting}

The most appropriate assessment tool will depend on a number of factors, such as the setting of the consultation (community or secondary care), focus of the consultation - (usual PD care or SPC), patient characteristics (i.e., presence of significant cognitive impairment), service configuration (integrated or prognostic gateway to palliative care), goal of assessment (clinical care or research) and the time available.

This article will focus on: (I) the needs assessments tools for PwP, rather than HRQoL assessment, (II) the characteristics of each tool and (III) suggest how they may fit within the overall assessment of unmet need within an integrated PD palliative care service. Although symptom specific measures are available, these lie outside the scope of this review which focuses on wider needs assessment.

We used a rapid review methodology. A single reviewer (ER) screened retrieved titles and abstracts from a limited number of electronic databases (Pubmed). The search terms "Parkinson(s)", "Palliat.", "Assess." were used PubMed. In addition, we drew on grey literature $(\mathrm{PhD}$ "Palliative care in Parkinson's disease: development of a needs assessment tool” Edward Richfield, University of Hull) to perform a 
narrative review of the existing palliative care assessment tools for use in $\mathrm{PwP}$.

\section{Generalised palliative assessment in PD}

\section{Palliative outcome score-symptoms-PD (POS-S PD)}

A common approach to the development of palliative needs assessment tools for PD has been to adapt existing tools, originally developed for other areas of palliative care. The POS (23) is a well-established palliative assessment originally validated in malignant conditions and subsequently in non-malignant conditions including MS (24). The core POS contains 10 items and forms the basis for a number of extended tools, such as the POS-S, where question 2 "other symptoms" is extended by adding a list of symptoms relating to the condition in question. A version of the POS-S has been independently validated for MS (POSS-MS) (24) and one was subsequently developed for PD (POS-S-PD) (25). Two publications, one cross sectional (25) and the other longitudinal (26), report the use of the POS$\mathrm{S}-\mathrm{PD}$ to assess palliative and supportive care need in $\mathrm{PwP}$ and atypical parkinsonism. Each paper refers to the tool with a different name - POS-PD and POS-PP respectively, but this appears to represent a single tool, which is now available for use on the host website as POS-S-PD.

There has been no reported independent validation of the POS-S-PD, although the core tool on which it is based has been well validated in a number of languages and the principle of extension to POS-S well described and validated in other conditions.

The POS-S-PD uses 20 questions to cover a range of motor and non-motor features of PD, each rated by the patient as 0 (no effect) to 4 (overwhelming), giving maximum total score of 80 .

The Core-POS covers 10 palliative care domains (pain, other symptoms, patient anxiety, family anxiety, information, support, depression, self-worth, wasted time, practical matters) again scored 0-4, maximum score 40.

The tools have been used in a cross-sectional study to describe symptom burden associated with later stage disease $(\mathrm{H}+\mathrm{Y} 3-5)(25)$. The study population was relatively young (mean age 67 at mean disease duration 9.7 years), given the average age of diagnosis in PD of 70 years. This is most likely to be reflective of a tertiary care centre study setting in South East England. People were excluded if care home resident, which is important given the high rates of care home use in later stage PD (27).
The authors present the scores for Core-POS (10 questions rated $0-4$, total score 40$)$ and POS-S-PD (20 questions $0-4$, total score 80 ) as separate but complementary assessments.

In combination these tools cover a broad range of potential palliative needs, focused predominantly on the person with PD, with just a single question (family anxiety) relating to other members and no explicit assessment of caregiver needs. They can be completed on the basis of patient or proxy report, which is an important strength.

A key strength of this assessment is the demonstrated responsiveness to change over time, with three assessments covering a $12-15$ month period, in a group with identical baseline characteristics (mean age 67, H+Y 3-5) (26).

\section{IPOS neuro/IPOS NEURO-8}

The IPOS NEURO (28) is a development of the POS and its related tools, specifically aimed at needs assessment in neurological conditions. It asks participants about their need in the three days prior to assessment. It starts with a prompt for main concerns (free text), followed by 34 symptom related questions, a prompt for other symptoms (free text) and 8 questions relating to other aspects of need (anxiety in patient and family, depression, peacefulness, ability to share with friends, information, practical issues, wasted time).

Eight of the symptom domains (pain, spasms, shortness of breath, nausea, vomiting, mouth problems, difficulty in sleeping, constipation), were combined in a symptom subscale [IPOS NEURO-8 (28)] and subjected to psychometric testing in patients with Multiple Sclerosis, PD, Progressive Supra-nuclear Palsy and Multiple System Atrophy.

Construct validity and test-retest reliability for the 8 question sub-scale (IPOS NEURO 8) was examined using a pooled data set taken from two independent studies, an intervention trial in MS and the longitudinal description of symptoms in PD and Related Conditions (PDRC) described above-data presumably extracted from the POS-S-PD scores. The patient characteristics for those with PDRC is therefore identical to above $(\mathrm{H}+\mathrm{Y} 3-5$, mean age 68 , care home residents excluded).

Test-Retest at 6 weeks was judged "fair" based on Pearson's correlation coefficient ( $\mathrm{r}=0.58)$.

Constructs showed moderate levels of validity against the PDQ-8 $(\mathrm{r}=0.48, \mathrm{P}<0.05)$, Non-Motor Symptom Questionnaire $(\mathrm{r}=0.58, \mathrm{P}<0.05)$ and Core POS $(\mathrm{r}=0.58, \mathrm{P}$ value not reported).

It is difficult to judge the external validity of these 
findings currently. As a standalone tool the IPOS NEURO-8 has reasonable psychometric properties, although limited by the study population which is not representative of $\mathrm{PwP}$ at either end of the disease course, (excluding $\mathrm{H}+\mathrm{Y}$ stage 1 and 2, at one extreme and care home residents at the other). The, as-yet un-reported, IPOS NEURO (only available as supplementary information to publication discussed above), containing the IPOS NEURO-8 as a subscale, would be useful as it covers a wide range of unmet need, including social and psychological domains.

\section{IPOS Neuro-s24}

The same group have recently published a factor analysis of a further assessment tool, the IPOS NEURO-s24 (29), which consist of 24 symptom-based questions, including those examined above in the IPOS NEURO-8 publications. The full version of this is not available currently, so it is unclear whether social, spiritual and caregiver domains are covered in addition to the 24 symptoms domains. Further publications are anticipated.

\section{ESAS-PD}

The ESAS-PD (2) is an adaptation of the Edmonton Symptom Assessment Scale (ESAS) (30), with the addition of $4 \mathrm{PD}$ related domains in addition to the original 10 item tool. The modifications were made following a modified Delphi process (3 movement disorder specialists), the tool asks respondents (patient $+/$ - help from caregiver) to rate the following on a 10 point scale: Pain, Tiredness, nausea, depression, anxiety, drowsiness, anorexia, wellbeing, shortness of breath, other problem, stiffness, constipation, dysphagia and confusion. Maximum score is therefore 140 .

Domains covered by the ESAS-PD relate to patient, rather than caregiver, experience. It is relatively short, reducing participant burden when compared with the POS$\mathrm{S}-\mathrm{PD} /$ core POS combination (30 questions).

The ESAS-PD is unique as the only tool in this area with published evidence to show responsiveness to intervention. Patients in a study mapping unmet need showed a significant reduction in ESAS-PD score after a period of intervention at a multi-disciplinary clinic.

Similar to the POS, described above, the original ESAS has been widely validated in malignant and some nonmalignant conditions (31-33). However, we were unable to identify any separate validation studies of the ESAS-
PD. Although there is evidence for change in response to intervention, the test-retest and inter-rater reliability to ESAS-PD (as opposed to ESAS) are not established. Also, whilst measuring presence and severity of symptom, it does not provide a patient priority with regard to which are the most concerning.

Since its development the ESAS-PD has been used to describe palliative care burden in other PD cohorts (34) and is included in current study protocols in this area (35).

\section{Palliative care assessment (PACA) tool}

PACA tool was developed in cancer as a way to rate the severity of patient identified symptoms (36). It was used to evaluate the symptom load of $\mathrm{PwP}$ in an incident cohort in the north of England (1). Participants self-generated a list of symptoms and were then prompted regarding additional 35 common symptoms including motor and non-motor. Each symptom then rated as 0 (not present) to 3 (present and dominating the day).

As individuals rated a variable number of symptoms, depending on how many they self-generated there is no summative score.

The tool was used in PwP but excluded those with atypical syndromes. The population was representative, including all $\mathrm{H}+\mathrm{Y}$ stages, with median age 75.4 years. Of the 161 patients 38 did not take part and were more likely to be older, in nursing homes or with more advanced disease, possibly reflecting the relatively high completion burden of this tool.

The pooled symptoms generated from the assessments were examined using factor analysis. Of the 11 factors identified six showed correlation with the Unified Parkinson's Disease Rating Score (UPDRS) ( $\mathrm{r}=0.33-0.58$ ). Reliability characteristics were not examined.

The administration time was not recorded, but selfevidently varied according to the number of symptoms generated, the largest recorded being 29 individual symptoms.

\section{NAT:Parkinson's disease}

The Needs Assessment Tool Progressive Disease: Cancer (NAT:PD-C) was developed in Australia for use in cancer (37) and subsequently adapted and validated in non-malignant conditions $(38-40)$. It has been adapted for use in PD (NAT:PD) $(41,42)$.

It differs from the tools described above in several key 
areas. It is the only clinician completed tool in this group, it does not produce a quantitative summary score, but rather each domain is rated for degree of concern (0 no concern - 2 significant concern). Where concerns are identified users are prompted to triage according to whether the unmet need can be addressed by themselves, someone in their team, or requires external referral (including, but not exclusive to, SPC referral). In addition, it contains at the start a prompt to identify $\mathrm{PwP}$ at higher risk of unmet needs and supplies suggested phrases to use to probe domains for those less familiar with this approach.

The NAT:PD contains 13 constructs within 3 domains (1-patient wellbeing, 2-ability of carer to care for patient, 3 -carer and family wellbeing). Constructs cover physical, psychological, spiritual and existential, financial and interpersonal aspects of needs, as well as rating information needs. As such it may be considered the most holistic if the tools discussed in this section, although similar domains are covered by the proposed IPOS NEURO. However, NAT:PD is a clinician-rated needs assessment tool and not an outcome measure, and the IPOS NEURO is patient or clinician rated and has the properties of an outcome measure, and could be used in conjunction with the NAT:PD.

Construct validation of the NAT:PD was conducted in a district general hospital population in the UK, with all disease stages represented $(\mathrm{H}+\mathrm{Y} 1-5)$. However, unlike the POS-S-PD and IPOS NEURO 8, the validation study did not include people with atypical parkinsonian syndromes.

Of the constructs tested (financial needs were not tested as no appropriate comparator identified), the level of correlation between each construct and the primary comparator tool was highly statistically significant for most constructs. The authors report good levels of agreement $(>0.6)$ for two constructs, moderate $(0.4-0.6)$ for five constructs (and fair (0.2-0.4) for four. Lower levels of agreement are explained by difficulty matching comparator tools to the broad constructs. Validity is further supported by the presence of convergent and divergent patterns of association.

Inter-rater reliability was assessed using videoed, NAT:PD guided, consultations. Reliability was good for one construct, moderate for three constructs and fair for six constructs. Three constructs failed to demonstrate at least fair agreement, although in two of these cases the level of percentage agreement was extremely high. This is an anomaly of Kappa, which can give poor agreement where percentage agreement is very high but not perfect (43), and the authors suggest Kappa be interpreted alongside percentage agreement in this instance.

The NAT:PD has previously been questioned for the time taken for administration (44). This was not assessed in the validation studies. However, it is designed for use by clinicians as part of routine consultation. Studies of the parent tool, in cancer, showed no increase in consultation time in an oncology clinic (45). It could also be used in conjunction with a patient reported tool such as the IPOS NEURO 8, which would highlight the most pressing concerns of the patient, thereby streamlining the consultation to focus on the most important issues.

\section{Caregivers}

Where informal caregivers are present PD can be associated with significant caregiver strain (46), which is particularly associated with the non-motor components of the disease (47), increases with disease severity and may affect younger carers to a greater degree than older ones (48). Caregiver burden has been the subject of a recent comprehensive systematic literature review which included a systematic review of available assessment tools (49). Here we summarise the characteristics of one instrument validated in $\mathrm{PD}$ and another identified by the review as being used most commonly in studies involving $\mathrm{PwP}$.

\section{Zarit burden interview (ZBI)}

ZBI (50) consists of 22 items examining caregiver burden, each scored 0 (never) -4 (almost always), with a total score out of 88 and a score of 21 felt to represent a threshold for significant burden. The full form has been validated in PD (51) with constructs achieving satisfactory levels of correlation $(\mathrm{r}=0.31-0.78)$.

These results have been closely replicated in a further study of $\mathrm{PwP}$ (52), which also examined the criterion validity of various short forms of the ZBI (Zarit 12, 8, 7, 6, 4 and 1), where the ZBI 22 was taken as the criterion gold standard. This latter aspect may require some caution in interpretation as scores for the short forms were derived from the original 22 item questionnaire, rather than separate administration. However, the evidence of good criterion validity ( $r=0.87-0.99)$ open up the opportunity to use shortened version of the $\mathrm{ZBI}$ in $\mathrm{PD}$, the author suggesting that shorter forms may be appropriate for screening or where burden is not the central variable under examination. 
Table 1 Comparison of general palliative care assessments for PDRD

\begin{tabular}{|c|c|c|c|c|c|c|c|c|}
\hline $\begin{array}{l}\text { Name of palliative } \\
\text { assessment tool }\end{array}$ & $\begin{array}{l}\text { Disease } \\
\text { stage in } \\
\text { which tool } \\
\text { tested }\end{array}$ & $\begin{array}{l}\text { Construct } \\
\text { validity }\end{array}$ & $\begin{array}{l}\text { Test-retest } \\
\text { reliability }\end{array}$ & $\begin{array}{l}\text { Inter rater } \\
\text { reliability }\end{array}$ & $\begin{array}{c}\text { Tested in } \\
\text { atypical } \\
\text { Parkinsonian } \\
\text { syndromes }\end{array}$ & $\begin{array}{c}\text { Respondent } \\
\text { (patient/caregiver/ } \\
\text { both/clinician only) }\end{array}$ & $\begin{array}{l}\text { Established } \\
\text { clinically } \\
\text { significant } \\
\text { change }\end{array}$ & $\begin{array}{c}\text { Burden of completion } \\
\text { (time/no. questions) }\end{array}$ \\
\hline POS-S PD & $\mathrm{H}+\mathrm{Y} 3-5$ & No & No & No & Yes & $\begin{array}{c}\text { Patient (core-POS } \\
\text { either patient or } \\
\text { carer) }\end{array}$ & - & $\begin{array}{c}20+10 \\
\text { (core POS) }\end{array}$ \\
\hline IPOS Neuro 8 & $\mathrm{H}+\mathrm{Y} 3-5$ & Yes & Yes & No & Yes & Patient & No & 8 \\
\hline PACA & $\mathrm{H}+\mathrm{Y} 1-5$ & Yes & No & No & No & Patient & No & Variable \\
\hline $\begin{array}{l}\text { NAT:Parkinson's } \\
\text { disease }\end{array}$ & $\mathrm{H}+\mathrm{Y} 1-5$ & Yes & No & Yes & No & Clinician & $N / A^{*}$ & $\begin{array}{l}13 \text { (no additional } \\
\text { burden to patient) }\end{array}$ \\
\hline
\end{tabular}

*, NAT: Parkinson's disease is not an outcome measure, rather gives a profile of concerns and triaged actions. POS-S PD, palliative outcome score-symptoms-PD; ESAS PD, Edmonton Symptom Assessment Score PD; IPOS Neuro 8, Integrated Palliative Outcome Scale neuro 8; PACA, palliative care assessment; POS, palliative outcome score; PDRD, Parkinson's disease and related disorders.

\section{Parkinson's disease caregiver burden (PDCB) questionnaire}

The PDCB has been developed specifically for PD, based on interviews with $\mathrm{PwP}$ and their caregivers (53). It consists of 20 items covering seven domains (Physical burden, sleep disruption, patient symptoms, responsibilities, medication (patient), social burden, patient and self-relationship).

Face and content validity were checked using patient groups and experts in the field. Internal consistency was good (Cronbach's alpha 0.856) although the physical burden item was felt to be less reliable and contribute poorly to over-all score.

Construct validity, examined against the Caregiver Burden Index (CBI), for over-all score was very good $(\mathrm{r}=0.773, \mathrm{P}<0.01)$. This may be anticipated as both tools explicitly measure the same variable (caregiver burden), in contrast with the tools discussed above. It should be noted that the CBI has not been independently validated in PD.

An alternative approach to measuring the impact of care on caregivers is to examine caregiver quality of life (QoL). Whilst this is not within the remit of the current article, it should be noted that tools to examine caregiver QoL have been specifically developed for PD (54) and atypical parkinsonism (55).

\section{Assessment tools in practice}

In practice, the choice of assessment tool (see Table 1) will depend on the model of palliative care being delivered and the characteristics of the person being assessed. The nature of PD means that tools which allow proxy or clinician assessment, rather than relying solely on patient reporting, will have an important role. Conversely, patient reported tools, particularly those such as the PACA, can help to prioritise patient concerns within an over-all assessment of needs.

In addition, the timing of the consultation, within an over-arching model of care, will determine the time available and the type of assessment which is most appropriate (see Figure 1). Thus, it is likely that a combination of tools, at different points in the care model and according to individual participant characteristics will be necessary.

\section{The challenge of palliative assessment}

Several studies suggest a significant burden of unmet palliative care needs in PD $(1,2,56)$, which may currently be invisible to clinicians. The application of structured assessments to $\mathrm{PwP}$ is therefore likely to reveal levels of palliative need which current services may not be equipped to meet. In addition, the need for education, training and support for clinicians, and commitment by service providers and commissioners must not be under-estimated. Implementation work with the Needs assessment Tool versions for interstitial lung disease (39) and heart failure (38) have both shown that usual care teams need training in 


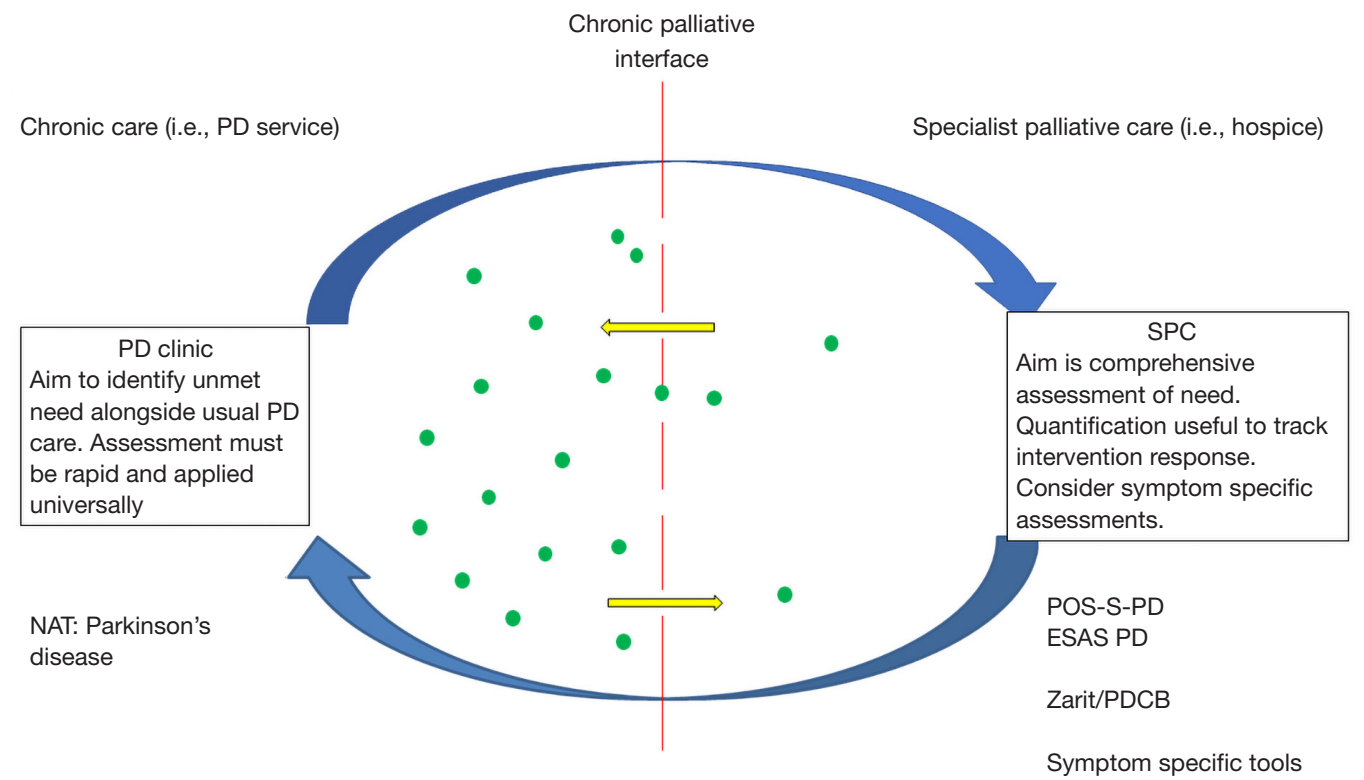

Figure 1 Potential use of tools within integrated delivery of palliative care for Parkinson's disease (PD). POS-S PD, palliative outcome score-symptoms-PD; ESAS PD, Edmonton Symptom Assessment Score PD; PDCB, Parkinson's disease caregiver burden; SPC, specialist palliative care.

how to identify, assess and manage symptoms, how to assess psychosocial concerns, how to communicate well with regard to ACP, how to raise the subject of SPC referral and how to develop robust clinical pathways of care including SPC $(57,58)$.

The growing evidence identifying predictors of poor prognosis in PD and the future possibility of individualised prognostication from baseline characteristics $(17,18)$, also present a significant challenge and are likely to increase the need for supportive interventions earlier in the disease course. We need to develop services which acknowledge and address these challenges. Through research and service evaluation, assessment tools can help us to estimate the volume of unmet need, identify priority areas for individual and service level development, target clinical and educational resource and assess the effectiveness of our interventions. As such they are a key component of patient focused care.

\section{Conclusions}

A number of palliative care assessment tools have been developed or adapted for use in PD. These vary greatly in the domains covered, the nature of respondent, burden of administration and the degree to which they have been formally subjected to psychometric testing. Choice of tool will depend upon the setting in which it is to be applied, the time available and the stage of disease. The rising rate of cognitive impairment in latter stage PD requires particular consideration and increases the utility of tools which accommodate proxy and clinician responses.

More than one tool may be needed to achieve a systematic, holistic assessment of need, perhaps applied at different points in the assessment process, with different purposes (identification, detailed assessment, quantification and response to change). Examining how these tools can supplement each other in practice and, most importantly, improve patient focused outcomes will be an important area of future research.

\section{Acknowledgments}

None.

\section{Footnote}

Conflicts of Interest: The authors have no conflicts of interest to declare.

Ethical Statement: The authors are accountable for all aspects of the work in ensuring that questions related 
to the accuracy or integrity of any part of the work are appropriately investigated and resolved.

\section{References}

1. Lee MA, Prentice WM, Hildreth AJ, et al. Measuring symptom load in Idiopathic Parkinson's disease. Parkinsonism Relat Disord 2007;13:284-9.

2. Miyasaki JM, Long J, Mancini D et al. Palliative care for advanced Parkinson disease: an interdisciplinary clinic and new scale, the ESAS-PD. Parkinsonism Relat Disord 2012;18 Suppl 3:S6-9.

3. World Health Organisation. WHO definition of palliative care 2015. Available online: http://www.who.int/cancer/ palliative/definition/en/

4. Parkinson's disease in Adults [Internet]. 2017 [cited 25/10/17]. Available online: https://www.nice.org.uk/ guidance/NG71

5. Giles S, Miyasaki J. Palliative stage Parkinson's disease: patient and family experience of health care services. Palliat Med 2009;23:120-5.

6. Tuck KK, Brodd L, Nutt J et.al. Preferences of patients with Parkinson's disease for communication about advanced care planning. Am J Hosp Palliat Care 2015;32:68-77.

7. Richfield EW, Alty J, Jones E. Palliative care for Parkinson's disease: A summary of evidence and future directions. Palliat Med 2013;27:805-10.

8. National Council for Palliative Care. Palliative care explained 2015. Available online: http://www.ncpc.org.uk/ palliative-care-explained

9. Currow DC, Allingham S, Yates $\mathrm{P}$, et al. Improving national hospice/pallative care service symptom outcomes systematically through point-of-care data collection, structured feedback and benchmarking. Support Care Cancer 2015;23:307-15.

10. Richardson A, Medina J, Brown V, et al. Patients' needs assessment in cancer care: a review of assessment tools. Support Care Cancer 2007;15:1125-44.

11. Homsi J, Walsh D, Rivera N, et al. Symptom evaluation in palliative medicine: patient report vs systematic assessment. Support Care Cancer 2006; 14:444-53.

12. Walsh RI, Mitchell G, Francis L, et al. What Diagnostic tools exist for the early identification of Palliative care Patients in General Practice? A systematic review. J Palliat Care 2015;31:118-23.

13. Datla S, Verberkt C, Hoye A, et al. Multi-disciplinary palliative care is effective in people with symptomatic heart failure: a systematic review and narrative synthesis. Palliat Med 2019;33:1003-16.

14. Maas EA, Murray S, Engels Y et.al. What tools are available to identify patients with palliative care needs in primary care: a systematic literature review and survey of European practice. BMJ Support Palliat Care 2013;3:444-51.

15. Thomas K. editor. The Gold Standards Framework Prognostic Indicator Guidance. 4th ed. UK: Shrewsbury; 2011.

16. Highet G, Crawford D, Murray SA, et al. Development and evaluation of the Supportive and Palliative Care Indicators Tool (SPICT): a mixed-methods study. BMJ Support Palliat Care 2014;4:285-90.

17. Macleod AD, Dalen I, Tysnes O, et al. Development and validation of prognositic survival models in newly diagnosed Parkinson's disease. Mov Disord 2018;33:108-16.

18. Velseboer DC, de Bie R, Wieske L, et al. Development and external validation of a prognostic model in newly diagnosed Parkinson disease. Neurology 2016;86:986-93.

19. Hely MA, Reid WG, Adena MA, et al. The Sydney multicenter study of Parkinson's disease: the inevitability of dementia at 20 years. Mov Disord 2008;23:837-44.

20. Aarsland D, Anderson K, Larsen JP, et al. Prevalence and characteristics of dementia in Parkinson's disease: an 8 year prospective trial. Arch Neurol 2003;60:387-92.

21. Sebring K, Shattuck J, Berk J, et al. Assessing the validity of proxy caregiver reporting for potential palliative care outcome measures in Parkinson's disease. Palliat Med 2018;32:1522-8.

22. Colagiuri B, King M, Butow J, et al. A comparison of the FACT-G and the Supportive Care Needs Survey (SCNS) in women with ovarian cancer: unidimensionality of constructs qual life res. Qual Life Res 2012;21:887-97.

23. Hearn J, Higginson I. Validation of core outcome measure for palliative care - the palliative care outcome scale (POS). Palliat Med 1998;12:474.

24. Sleeman KE, Higginson I. A psychometric validation of two brief measures to assess palliative need in patients severely affected by multiple sclerosis. J Pain Symptom Manage 2013;46:406-12.

25. Saleem TZ, Higginson IJ, Chaudhuri KR, et al. Symptom prevalence, severity and palliative care needs assessment using the Palliative Outcome Scale: a cross-sectional study of patients with Parkinson's disease and related neurological conditions. Palliat Med 2013;27:722-31.

26. Higginson IJ, Gao W, Saleem TZ, et al. Symptoms 
and quality of life in late stage Parkinson syndromes: a longitudinal community study of predictive factors. PLoS One 2012;7:e46327.

27. Porter B, Henry S, Gray WK, et al. Care requirements of a prevalent population of people with idiopathic Parkinson's disease. Age Ageing 2010;39:57-61.

28. Gao W, Crosby V, Wilcock A, et al. Psychometric properties of a generic, patient-centred palliative care outcome measure of symptom burden for people with progressive long term neurological conditions. PLoS One 2016;11:e0165379.

29. Wilson R, Hepgil N, Sara RA, et al. symptom dimensions in people affected by long-term neurological conditions: a factor analysis of a patient-centred palliative care outcome symptom scale. Sci Rep 2019;9:4972.

30. Bruera E, Kuehn N, Miller MJ, et al. The Edmonton Symptom Assessment System (ESAS): a simple method of the assessment of palliative care patients. J Palliat Care 1991;7:6-9.

31. Savard J, Ivers H, Savard MH. Capacity of the Edmonton Symptom Assessment System and the Canadian Problem Checklist to screen clinical insomnia in cancer patients. Support Care Cancer 2016;24:4339-44.

32. Hui D, Bruera E. The Edmonton Symptom Assessment System 25 Years Later: Past, Present, and Future Developments. J Pain Symptom Manage 2017;53:630-43.

33. Davison SN, Jhangri G, Johnson JA. Cross-sectional validity of a modified Edmonton symptom assessment system in dialysis patients: a simple assessment of symptom burden. Kidney Int 2006;69:1621-5.

34. Tuck K, Mann L, Borcich T, et al. A Novel Interdisciplinary Palliative Care Clinic for Advanced Parkinson's Disease and Parkinsonian Syndromes. Neurology 2016;86:6.

35. Lennaerts H, Groot $M$, Steppe $M$, et al. Palliative care for patients with Parkinson's disease: study protocol for a mixed methods study. BMC Palliat Care 2017;16:61.

36. Wenham S, Gomm S. Palliative care assessment (PACA) tool - an effective clinicl outcome measure for a hospital palliative care team. Palliat Med 2006;20:151.

37. Waller A, Girgis A, Lecathelinais C, et al. Validity, reliability and clinical feasibility of a Needs Assessment Tool for people with progressive cancer. Psychooncology 2010;19:726-33

38. Waller A, Girgis A, Davidson P. Facilitating needs-based support and palliative care for people with chronic heart failure: preliminary evidence for the acceptability, interrater reliability, and validity of a needs assessment tool. J
Pain Symptom Manage 2013;45:912-25.

39. Johnson MJ, Jamali A, Ross J, et al. Psychometric validation of the Needs Assessment Tool: Progressive Disease in Interstitial Lung Disease. Thorax 2018;73:880-3.

40. Boland JW, Reigada C, Yorke J, et al. The Adaptation, Face, and Content Validation of a Needs Assessment Tool: Progressive Disease for People with Interstitial Lung Disease. J Palliat Med 2016;19:549-55.

41. Richfield EW, Girgis A, Johnson M. Assessing palliative care in Parkinson's disease-development of the NAT: Parkinson's disease. Palliat Med 2014;28:641.

42. Richfield E, Jones E, Johnson M. Validation Of A Screening Tool For Identifying Unmet Palliative Care Needs In Parkinson's Disease. Irish Ageing Studies Review 2015;6:103.

43. Bland M. Clinical measurement. An introduction to medical statistics. 4th ed. Oxford: Oxford University Press; 2015, 310 .

44. Kluger BM, Fox S, Timmons S, et al. Palliative care and Parkinson's disease: Meeting summary and recommendations for clinical research. Parkinsonism Relat Disord 2017;37:19-26.

45. Waller A, Girgis A, Johnson C, et al. Implications of a needs assessment intervention for people with progressive cancer: impact on clinical assessment, response and service utilisation. Psychooncology 2012;21:550-7.

46. Peters M, Fitzpatrick R, Doll H, et al. Does self-reported well-being of patients with Parkinson's disease influence caregiver strain and quality of life? Parkinsonism Relat Disord 2011;17:348-52.

47. Carter JH, Stewart BJ, Lyons KS, et al. Do motor and nonmotor symptoms in $\mathrm{PD}$ patients predict caregiver strain and depression? Mov Disord 2008;23:1211-6.

48. Carter JH, Lyons KS, Stewart BJ, et al. Does age make a difference in caregiver strain? Comparison of young versus older caregivers in early-stage Parkinson's disease. Mov Disord 2010;25:724-30.

49. Mosley PE, Moodie R, Dissanayaka N. Caregiver Burden in Parkinson Disease: A Critical Review of Recent Literature. J Geriatr Psychiatry Neurol 2017;30:235-52.

50. Zarit SH, Reever KE, Bach-Paterson J. Relatives of the Impaired Elderly: Correlates of Feelings of Burden. Gerontologist 1980;20:649-55.

51. Martinez-Martin P, Forjaz MJ, Frades-Payo B, et al. Caregiver burden in Parkinson's disease. Mov Disord 2007;22:924-31; quiz 1060.

52. Hagell P, Alvariza A, Westergren A, et al. Assessment 
of Burden Among Family Caregivers of People With Parkinson's Disease Using the Zarit Burden Interview. J Pain Symptom Manage 2017;53:272-8.

53. Zhong M, Evans A, Peppard R, et al. Validity and reliability of the PDCB: a tool for the assessment of caregiver burden in Parkinson's disease. Int Psychogeriatr 2013;25:1437-41.

54. Jenkinson C, Dummett S, Kelly L, et al. The development and validation of a quality of life measure for the carers of people with Parkinson's disease (the PDQ-Carer). Parkinsonism Relat Disord 2012;18:483-7.

55. Pillas M, Selai C, Quinn NP, et al. Development and validation of a carers quality-of-life questionnaire for parkinsonism (PQoL Carers). Qual Life Res 2016;25:81-8.
56. Hudson PL, Toye C, Kristjanson LJ. Would people with Parkinson's disease benefit from palliative care? Palliat Med 2006;20:87-94.

57. Reigada C, Papadopoulas A, Boland JW, et al. Implementation of the Needs Assessment Tool for patients with interstitial lung disease (NAT:ILD): facilitators and barriers. Thorax 2017;72:1049-51.

58. Janssen DJ, Boyne J, Currow DC, et al. Timely recognition of palliative care needs of patients with advanced chronic heart failure: a pilot study of a Dutch translation of the Needs Assessment Tool: Progressive Disease - Heart Failure (NAT:PD-HF). Eur J Cardiovasc Nurs 2019;18:375-88.
Cite this article as: Richfield EW, Johnson MJ. Palliative care in Parkinson's disease: review of needs assessment tools. Ann Palliat Med 2020;9(Suppl 1):S6-S15. doi: 10.21037/ apm.2019.09.18 